\title{
ELECTRONIC PROPERTIES OF TWO ISOMERIC CHARGE TRANSFER [2.2]PARACYCLOPHANES
}

\author{
D. SCHWEITZER, K.H. HAUSSER, V. TAGLIEBER and H.A. STAAB \\ Max-Planck-Institute, Depariment of Molecular Physics, Deparment of Organic Chemisrry. \\ 6900 Heideiberg, Jahn-Srrasse 29, FRG
}

Received 1 December 1975

\begin{abstract}
The emission specura and the zero field splitting parameters of the two diastereomeric 4,7-dicyano-12,15-dime thoxy12.2] piracyclophanes 3 and 4 in comparison to the co:responding monomers 1 and 2 were investigated in order to study the orientation dependence of charge transfer (CT) interactions. The general results in glasses (PMMA) are: broad structureless emission bands with large spectral overlap between fuorescence and phosphorescence; strong reduction of the zera field splitting parameters $D$ and $D^{*}$ by a factor of two for the pseudo-ortho isomer 3 and by a factor of four for the pseudogeminal isomer 4 showing the strong effect of the geometrical orientaticn. In single crystals of the same phanes the zero Field parameters were found to be further reduced to about one fifth of the value of the monomers which indicates, in accordance with the emission spectra, an additional intermolecular interaction between adjacent phanes.
\end{abstract}

\section{Introduction}

The optical absorption and emission spectra of charge-transfer (CT) complexes has been the subject of extensive research over more than 30 years. More recently, several papers also dealt with the ESR of the optically excited triplet states of such complexes $[1-6]$.

Characteristic features of CT complexes are broad structureless fluorescence and phosphorescence spectra which show a large spectral overlap [7]. Furthermore, the zero field splitting parameters $D$ and $E$ of the excited triplet states of CT complexes were found to be considerably smaller than those of the monomer donor and acceptor molecules forming the CT complexes [1-6].

One problem in comparing theoretical calculations on CT complexes with experimental results originates from the fact that for CT complexes in solution the precise geometrical orientation of the donor with respect to the acceptor is not known, neither is it known whether there is one unique or several different orientations. On the other hand, in a single crystal there are usually no donor-acceptor pairs. Furthermore, just one amangement exists and there is no way to vary the relative orientation of donor and acceptor in order to study geometrical effects. This drawback does not exist if the charge-transfer occurs intramolecularly in a [2.2] paracyclophane consisting of one donor and one acceptor in different well-defined rigid geometrical arrangements. For a number of such donor-acceptor paracyclophanes it was recently shown by our group that the strong orientation effects on the $\mathrm{CT}$ absorption exist [8].

Freviously, we investigated a series of naphthaleno-, biphenylo- and phenanthreno-[2.2]phanes consisting of two identical aromatic units $[9,10]$. The general results were broad emission bands with little or no structure shifted to the red by about $4000-8000 \mathrm{~cm}^{-1}$ and a decrease of the $D$ (and $E$ ) parameters of the order of 10 to $20 \%$ only with respect to the mono. mers. This indicates that the two unpaired electrons of the excited triplet state have a high probability to be at a given time in the same part of the molecule.

In this paper we report on the emission spectra and on the $D$ and $E$ parameters of the pseudo-ortho and the pseudo-gemirial isomers of the 4,7-dicyano-12,15dimethoxy [2.2] paracyclophane 3 and 4 which are compiled in fig. 1 together with the two monomers 1,4-dimethoxy-2,5-dimethylbenzene (1) and 1,4 dicyano-2,5-dimethylbenzene (2). 
<smiles>COc1cc(C)c(C(C)(C)C)cc1OC</smiles>

$\stackrel{1}{=}$

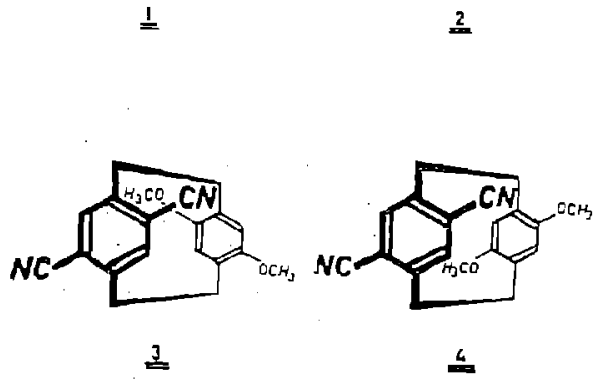

Fig. 1. 1: 1,4-dimethoxy-2,5-dimethylbenzene;

2: 1,4-dicyano-2,5-dimethylbenzene;

3: pseudo-or tho 4,7-dicyano-12,15-dime thoxy [2.2]paracyclophane;

4: pseudo-geminal 4,7-dicyano-12,15-dimethoxy[2,2]paracyclophane.

\section{Experiments}

The preparation of the two diastereomeric 4,7 dicyano-12,15-dimethoxy [2.2] paracyclophanes (3) and (4) will be described separately [11]. The glassy samples in polymethylmethacrylate (PMMA) with a concentration $c \leqslant 10^{-2} \mathrm{~mol} / \mathrm{l}$ of 2,3 and 4 were prepared as described previously [9], while the single crystals of $\mathbf{3}$ and $\mathbf{4}$ were obtained from a saturated solution in chloroform. Their size was in the range between 1 and $3 \mathrm{~mm}^{3}$. The donor 1 was measured in a glass matrix of $n$-octane because its first excited singlet and triplet states lie above the corresponding states of PMMA [12]. The apparatus used to record the luminescence and the ODMR spectra was similar to the one described by Zuclich et al. [13]. All measurements were nerformed at $1.3 \mathrm{~K}$.

\section{Results}

Fig. 2 shows on top the total emission spectra of 3 and 4 obtained in a PMMA glass matrix as well as those measured with single crystals and in the middle the phosphorescence spectra of the same samples taken with a rotur. The intensity of the phosphorescence was only a few percent of the total emission; hence the

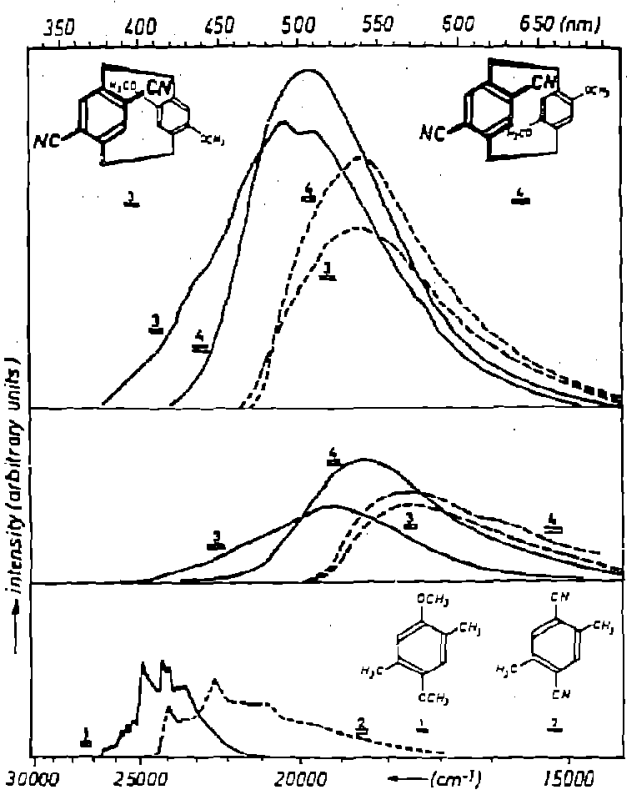

Fig. 2. Top: Total emission spectra of the two diastereomeric 4,7-dicyano-12,15-dimethoxy [2.2]paracyclophanes 3 and 4 in a PMMA glass matrix (-) and in single crystals (---) at $1.3 \mathrm{~K}$.

Middle: Phosphorescence spectra taken with a rotor of the same [2.2] paracyclophanes 3 and 4 in PMMA $(-)$ and in single crystals (---) at $1.3 \mathrm{~K}$.

Bottom: Phosphorescence spectra taken with a rotor of the monomer 1,4 -dimethoxy-2,5-dimethylbenzene ( $1 j$ in $n$-octane and of 1,4-dicyano-2,5-dimethylbenzene (2) in PMMA at $1.3 \mathrm{~K}$.

total emission spectra represent to a good approximation the fluorescence spectra of 3 and 4 . The phosphorescence spectra of 1 in $n$-octane and of 2 in PMMA are

Table 1

Red shifts of cmission spectra of [2.2]paracyclophones 3 and 4 as compared to the emission spectra of monomer 2 in PMMA

\begin{tabular}{|c|c|c|c|}
\hline \multirow[b]{2}{*}{$\begin{array}{l}\text { Type of } \\
\text { spectral shift }\end{array}$} & \multirow[b]{2}{*}{ Phane } & \multicolumn{2}{|l|}{ Matrix } \\
\hline & & $\begin{array}{l}\text { FMMA } \\
\left(\mathrm{cm}^{-1}\right)\end{array}$ & $\begin{array}{l}\text { Single crystal } \\
\left(\mathrm{cm}^{-1}\right)\end{array}$ \\
\hline Fluorescence & $\begin{array}{l}3 \\
4\end{array}$ & $\begin{array}{l}10600 \\
11500\end{array}$ & $\begin{array}{l}12600 \\
12800\end{array}$ \\
\hline Phosphorescence & $\begin{array}{l}3 \\
4\end{array}$ & $\begin{array}{l}4700 \\
5600\end{array}$ & $\begin{array}{l}6500 \\
6700\end{array}$ \\
\hline
\end{tabular}


Table 2

Microwave transitions and triplet zero field splitting parameters $|E|,|D|$, and $D^{*}$ of the monomers 1 and 2 and of the [2.2] paracyclophanes 3 and 4 in a glass matrix and also of 3 and 4 in single crystals

\begin{tabular}{|c|c|c|c|c|c|}
\hline Substance & Matrix & Microwave transitions (GMz) & $\mid E_{1}\left(\mathrm{~cm}^{-1}\right)$ & $|D|\left(\mathrm{cm}^{-1}\right)$ & $D^{*}\left(\mathrm{~cm}^{-1}\right)$ \\
\hline $\begin{array}{l}\text { 1,4-dimethoxy-2,5- } \\
\text { dimethylbenzene (1) }\end{array}$ & $n$-octane & $\begin{array}{l}2.2125 \pm 0.015 \\
2.3746 \pm 0.020 \\
4.5595 \pm 0.030\end{array}$ & 0.0369 & 0.1160 & 0.1324 \\
\hline $\begin{array}{l}\text { 1,4-dicyano-2,5- } \\
\text { dimethylbenzene ( } 2 \text { ) }\end{array}$ & PNIMA & $\begin{array}{l}0.9045 \pm 0.030 \\
3.2348 \pm 0.020 \\
4.140 \pm 0.030\end{array}$ & 0.0151 & 0.1229 & 0.1257 \\
\hline $\begin{array}{l}\text { pseudo-ortho } 4,7- \\
\text { dicyano-12,15-di- }\end{array}$ & PMMA & $\begin{array}{l}0.9023 \pm 0.050 \\
1.4748 \pm 0.050 \\
2.3737 \pm 0.050\end{array}$ & 0.01503 & 0.0642 & 0.0693 \\
\hline $\begin{array}{l}\text { methoxy [2.2] para- } \\
\text { cyclophane (3) }\end{array}$ & single crystal & $\begin{array}{l}0.4545 \pm 0.005 \\
0.5481=0.005 \\
1.0028 \pm 0.005\end{array}$ & 0.00758 & 0.0259 & 0.0290 \\
\hline $\begin{array}{l}\text { pseudo-geminal 4,7- } \\
\text { dicyano-12,15-di- }\end{array}$ & PMMA & $\begin{array}{l}0.2956=0.050 \\
0.7764=0.050 \\
1.0693=0.035\end{array}$ & 0.00493 & 0.0313 & 0.0324 \\
\hline $\begin{array}{l}\text { methoxy [2.2]para- } \\
\text { cyclophane (4) }\end{array}$ & single crystal & $\begin{array}{l}0.2553 \pm 0.005 \\
0.5643 \pm 0.005 \\
0.8195 \pm 0.005\end{array}$ & 0.00426 & 0.0231 & 0.0242 \\
\hline
\end{tabular}

plotted in the lower part of fig. 2. In order to facilitate comparison, the wave numbers for the red shifts of the emission spectra of the phanes 3 and $\mathbf{4}$ as compared to the monomer 2 with the lower lying first excited states are compiled in table 1.

The microwave transitions and the zero field splitting parameters $|D|$ and $|E|$ of the monomers 1 and 2 and of the paracyclophanes 3 and 4 in a glass matrix are compiled in table 2, as well as those obtained with single crystals of 3 and 4 . The errors are due to the linewidth of the microwave transitions which was between 30 and $100 \mathrm{MHz}$ in the glasses, but was only about $5-10 \mathrm{MHz}$ in the single crystals.

The values given for $D^{*}=\left(D^{2}+3 E^{2}\right)^{1 / 2}$ are unambiguous since they are not affected by the assignment of the microwave transitions. For calculating $|D|$ and $[E]$ it was assumed that the low frequency microwave transition corresponds to $2|E|$ which is true for the large majority of excited triplet states of aromatic molecules. Note that the only possible alternative assignment would lead to even smaller $|D|$.values for the [2.2] paracyclophanes 3 and 4 and hence would not change the conclusions.

\section{Discussion}

The broad structureless fluorescence and phosphorescence bands observed with the paracyclophanes 3 and 4 are typical for excimers in general and for CT bands in particular [7]. The characteristic electronic properties of the excited triplet states of CT complexes originate from the fact that the two triplet electrons have a high probability to be at a given time in two different orbitals which are separated in space, i.e., the one electron is in the highest occupied orbital of the donor and the other in the lowest unoccupied orbital of the acceptor. As a consequence of this a smaller value of the exchange integral results and hence a smaller decrease of the energy of the first excited triplet state with respect to the corresponding singlet state. Consequently, the phosphorescence is less shifted to the red than the fluorescence and a large spectral overlap between the two is observed.

The absolute red shifts of our CT paracyclophanes (3) and (4) are considerably larges than those observed in $C T$ complexes indicating a stronger transannular $\pi$ electron interaction in the CT phanes as compared to 
CT complexes. It originates from the closer distance between the two units of about $3 \AA$ as compared to about 3.5 \& typical for CT complexes.

The larger red shift of the pseudo-geminal paracyclophane 4 as compared to the pseudo-or tho paracyclophane 3 in PMMA and the still larger red shift observed with single crystals is very analogous to the behaviour of the $|D|$ values. This analogy extends even to the details that the difference between 3 and 4 is much smaller in the single crystals than in the PMMA matrix, inspite of the fact that the absolute shifts are larger in the crystals. We shall discuss this behaviour below in connection with the $D$ parameters which are still more sensitive to differences in the interaction than the emission spectra.

A second consequence of the separation in space of the two triplet electrons in the CT-phanes mentioned above is a smaller dipolar coupling between these two electrons and hence a considerable decrease of the $D^{*}$ and $D$ parameters with respect to the moinomers in distingtion to the [2.2] phanes consisting of two identical units $[9,10]$. This is born out by the experimental results compiled in table 2 .

The considerable smiller $D^{*}$ parameter measured for the pseudo-geminal phane 4 as compared to the pseudo-ortho phane 3 in PMMA and the corresponding difference in the CT character show the important in. fluence of the geometrical orientation of the donor with respect to the acceptor. The [2.2] paracyclophanes are particularly suitable for studying this influence be. cause of their well-defined rigid structure.

A quantitative relation for the CT character $X$ of the lowest excited triplet state of a CT complex was given by Hayashi and Nagakura [4]:

$\chi=\left(D^{*}-D_{\mathrm{A}}^{*}\right) /\left(D_{C \mathrm{~T}}^{*}-D_{\mathrm{A}}^{*}\right)$,

where $D^{*}$ and $D_{A}^{*}$ are the experimental values for the ccmplex and for the acceptor $\mathrm{A}$, respectively, and $D_{\mathrm{CT}}^{*}$ is the theoretical value for a complete transfer of one electron $(100 \% \mathrm{CT})$. It was assumed that $D_{\mathrm{A}}^{*}=D_{\mathrm{D}}^{*}$, where $D_{\mathrm{D}}^{*}$ is the experimental value for the donor $D$, an assumption which is valid to a good approximation in our case, as can be seen from table 2.

It was pointed out [14] that eq. (1) based on $D^{*}$ might give erroneous results since all $D^{*} \mathrm{~s}$ are positive by definition, while $D$ might become negative as well [15]. However, it is clear that an equation for $X$ analogous to (1) is correct when $D$ is used instead of $D^{*}$. It appears that the calculated values for $D_{\mathrm{CT}}$ are negative [15]. If $D$ is negative, the results obtained using $D^{*}$ or $D$ values tend to agree within the limits of accuracy, but if $D$ is positive, they may differ rather considerably. In our case, with a distance between the donor and the acceptor of about $3 \AA$ typical for [2.2]paracyclophanes, $D_{C T}$ is about $-0.03 \mathrm{~cm}^{-1}$ [15]. If we assume $D$ to be negative, we obtain a CT character $x$ of about 0.6 for the pseudo-ortho-[2.2] paracyclophane 3 and of about 1 for the pseudo-geminal phane (4). On the other hand, if $D$ is positive, the corresponding val. ues would be 0.4 for (3) and 0.6 for (4). We do not at tempt at this moment to give arguments for the sign of $D$ and hence for the absolute values of the CT character $\chi$, but we would rather like to emphasize that the ratio of $\chi_{\text {ortho }} / x_{\text {geminal }}$ is in any case about $2 / 3$ showing the importance of the geometrical orientation for the CT character.

Even smaller values for $D^{*}$ as well as for $|D|$ and $|E|$ are found when studying the 4,7-dicyano-12,15. dimethoxy[2.2] paracyclophanes in neat single crystals. We attribute this further reduction of the zero field splitting parameters to internolecular CT interactions between adjacent molecules in the single crystals because we cannot imagine any influence of the crystal which could change the intramolecular interactions so drastically as to produce a reduction of the zero field splitting parameters by more than a factor of two as is observed for the pseudo-ortho paracyclophane 3 in a neat single crystal as compared to the same phane in PMMA.

A detailed comparison of the $D^{*}$ and $D$ parameters of the two isomers in PMMA and in neat single crystals reveals the following result: While the values of $D^{*}$ and $|D|$ found for the pseudo-geminal phane 4 is PMMA are more than a factor of two smaller than those of the pseudo-or tho isomer 3 , the further reduction in the single crystals is much larger for the latter resulting in a considerably smaller difference of about $20 \%$ of the $D^{*}$ values of the two isomers in neat single crystals. Note the analogy of this observation with the red shifts of the emission lines discussed above.

The fact that the influence of the additional intermolecular interaction is smaller in the pseudo-geminal phane 4 than in the pseudo-ortho isomer 3 is rather plausible because the stronger intramolecular interaction leaves less room for an additional intermolecular interaction. It would Be tempting to interpret the 
large additional effects observed in a neat single crystal of the pseudo-ortho phane 3 by postulating a geometrical orientation between a donor and an acceptor unit of adjacent molecules in this crystal which favors intermolecular charge transfer interactions. In order to prove this postulated crystal structure, $X$-ray investigations of the single crystals of the two phanes 3 and 4 are under way.

The results of the emission spectra and of the zero freld splitting parameters seem to indicate that there is not much difference between the two isomers in single crystals. However, when investigating intersystem crossing rates and radiative and radiationless decay constants, properties of the system which depend mainly on intramolecular interactions, we found even in single crystals strong differences between the two isomers up to a factor of ten [16].

\section{Acknowledgement}

We are grateful to a referee for drawing our attention to the problems in connection with eq. (1) and to Professor J.P. Colpa for a discussion of the theoretical aspects.

\section{References}

[1] H. Hayashi, S. Nagakura and S. Ivata, Mol. Phys. 13 (1967) 489 .
[2] H. Beess, J. de Jone and A. Weller. Colloque Ampère 15 (1968) 289.

[3] H. Hayashi, S. Iwata and S. Nagakura, j. Chem. Phys50 (1969) 993.

[4] H. Hayashi and S. Nagakura, Mol. Phys. 19 (1970) 45.

[5] P. Krebs, E. Sackmann and J. Schwarz, Chem. Phys. Letters 8 (1971) 417.

[6] H. Möhwald and E. Sackmann, Chem. Phys. Letters 26 (1974) 509.

[7] S. Iwata, J. Tanaka and S. Nagakura, J. Chem. Phys. 47 (1967) 2203.

[8] H.A. Stagb and W. Rebafka, Angew, Chem. Intern. Edition 13 (1974) 203;

H.A. Staab, C. Herz and H.-E. Henke, Tetrahedron Letters (1974) 4393.

H.A. Stab and H. Haffner, ibid. 1974, 4397.

H. Vogler, G. Ege and H.A. Staab, Tetrahedron 3! (1975) 2441.

[9] D. Schweitzer, J.P. Colpa, J. Behnke, K.H. Hausser, M, Faenel and FLA. Stajb, Chem. Phys. 11 (1975) 373.

[10] D. Schweitzer, J.P. Colpa, K.H. Hausser, M. Haenel and H.A. Staab, J. Luminescence 12/13 (1976) 363.

[11] V. Taglieber and H.A. Staab, to be published.

[12] W.E. Graves, R.H. Hofeldt and S.P. MøGiyn, J. Chem. Phys. 56 (1972) 1309.

[13] J. Zuclich, D. Schwcitzer and A.H. Maki, Photochem. Photobiol. 18 (1973) 161.

[14] P. Krebs, Thesis, Stuttgart University (1973).

[15] J. de Jong, Thesis, Free University, Amsterdam (1970).

[16] D. Schweitzer, K.H. Hausser and H.A. Staab, to be published. 